\title{
Deconstructing the Complexity of TGF $\beta$ Signaling in Hematopoietic Stem Cells: Quiescence and Beyond
}

\author{
Ashwini Hinge ${ }^{1,2} \cdot$ Marie-Dominique Filippi ${ }^{1,2}$
}

Published online: 29 October 2016

(C) Springer International Publishing AG 2016

\begin{abstract}
The hematopoietic system is highly dynamic and must constantly produce new blood cells every day. Mature blood cells all derive from a pool of rare longlived hematopoietic stem cells (HSCs) that are mostly quiescent but occasionally divide and self-renew in order to maintain the stem cell pool and continuous replenishment of mature blood cells throughout life. A tight control of HSC self-renewal, commitment to differentiation, and maintenance of quiescence states is necessary for lifelong blood supply. Transforming growth factor- $\beta$ (TGF- $\beta$ ) is a critical regulator of hematopoietic cell functions. It is a potent inhibitor of hematopoietic cell growth. However, TGF $\beta$ functions are more complex and largely contextdependent. Emerging evidence suggests a role in aging, cell identity, and cell fate decisions. Here, we will review the role of TGF- $\beta$ and downstream signaling in normal HSC functions, in HSC quiescence, and beyond.
\end{abstract}

Keywords TGF $\beta$ - Hematopoietic stem cell - Quiescence · Fate determination

This article is part of the Topical Collection on Role of Classical Signaling Pathways in Stem Cell Maintenance

Marie-Dominique Filippi

Marie-Dominique.Filippi@cchmc.org

1 Division of Experimental Hematology and Cancer Biology - S7.605, Cincinnati Children's Research Foundation, 3333 Burnet Av, Cincinnati, OH 45229, USA

2 University of Cincinnati College of Medicine, Cincinnati, OH 45229, USA

\section{Introduction}

The hematopoietic system is a highly dynamic tissue that must constantly produce new blood cells every day owing to the relative short life-span of mature blood cells. The daily turnover of neutrophil production is $10^{10}-10^{11}$ per human body. This high cellular turnover requires a tightly orchestrated system. The hematopoietic system has long been viewed as a hierarchical system whereby the billions of short-lived mature blood cells all derive from a pool of rare long-lived hematopoietic stem cells (HSCs). The capacity of HSC to also selfrenew enables maintaining the stem cell pool and continuous replenishment of mature blood cells throughout life $[1,2]$. In this model, HSCs are mostly quiescent or very slow cycling, which is necessary for maintaining a life-long pool of HSC [3]. When they occasionally divide, they decide to generate a progeny that progressively loses self-renewal properties while acquiring highly proliferative capacity and committing to lineage differentiation, and/or another cell that retain stemness properties and returns into dormancy [4]. Therefore, the hematopoietic system is maintained by a well-orchestrated equilibrium between HSC quiescence, survival, self-renewal, and differentiation in order to sustain the regenerative needs of the hematopoietic tissue under homeostatic or stress conditions, while averting cell loss or cell overgrowth [1,2].

HSCs are located in a specialized bone marrow microenvironment, or 'niche', where they respond to a variety of signals emanating from soluble factors and surrounding cells [5-7]. One such factor is the transforming growth factor- $\beta$ (TGF $\beta$ ). The TGF $\beta$ family of cytokines encompasses the TGF $\beta$ /Activin/Nodal subfamily and the BMP (bone morphogenetic protein)/GDF (growth and differentiation factor)/MIS (Muellerian inhibiting substance) subfamily [8]. TGF $\beta$ proteins are pleiotropic factors and play fundamental roles during normal development, under 
regenerative conditions and in various diseases [8-11]. In the hematopoietic system, TGF $\beta$ is largely recognized as a potent inhibitory factor of hematopoietic cell growth. However, TGF $\beta$ functions are more complex and evidence suggests it can also promote cell growth and participate in lineage differentiation $[9,11]$. The plurality of TGF $\beta$ biological functions stems from a number of factors. TGF $\beta$ proteins are secreted by virtually every cell in the body, including hematopoietic cells, and can act in a paracrine or autocrine manner [12]. They signal though numerous signaling pathways, including canonical Smad transcription factors and non-smad signaling pathways [13], and interact with a number of developmental pathways such as Wnt, NOTCH, and Hippo [14], to control expression of target genes. Hence, TGF $\beta$ biological outcome varies depending on dosage, cell type, differentiation stage and environment [8]. There is little information on the role of BMPs in adult hematopoiesis. This review is thus focused on TGF $\beta$ proteins. We will deconstruct the complexity of TGF $\beta$ in normal adult hematopoietic stem cell functions.

\section{The TGF $\beta$ Signaling Pathway}

TGF $\beta$ factors (TGF $\beta 1,-2,-3$ ) are synthetized as proproteins that are associated with a propeptide also known as the latency associated protein (LAP), which renders the growth factor latent. In addition, the latent TGF- $\beta$ are secreted as a complex in which the latent TGF $\beta$ is bound to the latent TGF- $\beta 1$ binding protein-1 (LTBP1), forming a large latent complex. The binding to LAP and LTBP1 blocks the ability of TGF $\beta$ ligand to interact with the receptor, so it is named 'inactive' or latent TGF $\beta$. Dissociation from the complex is necessary for ligand access to their receptors and, therefore, TGF $\beta$ biological activity [15]. Several mechanisms for releasing TGF $\beta$ from the latent complex have been proposed. Latent TGF $\beta$ can be processed into bioactive form by proteolysis (mediated by elastases or metalloproteinases) $[16,17]$. TGF $\beta$ can also be activated by the matricellular protein thrombospndin-1, acting by disrupting interaction between LAP and TGF $\beta$ [18]. In addition, some integrins can increase the extracellular concentration of active TGF $\beta$ proteins. In this case, the LAP of TGF $\beta 1$ and TGF $\beta 3$ proproteins contains an integrin binding site (RGD domain) enabling their direct interaction with several types of integrin, including $\alpha \mathrm{V}$-containing integrins, $\alpha \operatorname{IIb} \beta 3, \alpha 5 \beta 1$, and $\alpha 8 \beta 1$. These interactions liberate the active protein from the latent complex by exertion of force on the integrin-binding domain [19]. Finally, both in vivo and in vitro evidence suggest $\mathrm{TGF} \beta$ can be activated by direct oxidation via reactive oxygen species and by mild acid treatment $[20,21]$.
TGF $\beta$ signals by binding to two membrane-bound serine/ threonine kinase receptors, type I and type II receptors, and trigger a variety of signaling pathways, including canonical Smad and non-Smad pathways [13, 22]. Upon ligand binding, the constitutively activated type II receptor (TGFBRII) recruits and activates by phosphorylation the type I receptor (TGFBRI). The activated TGFBRI, also known as ALK5, phosphorylates the Smad family members. There are three classes of Smad proteins, receptor-activated Smad (R-Smad), common-partner of Smad (Co-Smad), and inhibitory-Smad (I-Smad). TGF $\beta$ is known to activate Smad 2 and 3, whereas Activin leads to Smad1,5,8 activation. R-Smads then associate with the CoSmad 4 forming a stable complex that translocates to the nucleus for gene transcription. Alternatively, Smad2/3 can bind TIF $1 \gamma$ for gene expression regulation [23]. The I-Smad 7, which is up-regulated in response to TGF $\beta$ signaling, then terminates the signals by binding to and inhibiting TGFBRI. TGF $\beta$ can also signal via other signaling pathways. The best known non-smad pathways are the JNK and p38 cascades. TGF $\beta$ can activate tumor necrosis factor (TNF)-receptor-associated factor 6 (TRAF6) and TGF $\beta$ - associated kinase 1 (TAK1) [24], which then activate JNK via MKK4 and p38 via MKK3/6 [25]. TGF $\beta$ can also induce activation of ERK $^{\mathrm{MAPK}}$, phosphoinositide 3-kinase-Akt-mTOR pathway, and small Rho GTPases [22] (Fig. 1). Finally, TGF $\beta$ signaling crosstalks with many additional pathways, including WNT, HIPPO, NOTCH, Hedgehog PI3K, and ERK ${ }^{\mathrm{MAPK}}$, which contributes to the versatile and pleiotropic role of TGF $\beta$ in many cellular processes [26].

\section{The Conundrum of TGF $\beta$ Signaling: Quiescence or Not Quiescence?}

\section{TGF $\beta$ in HSC Quiescence}

In the hematopoietic system, TGF $\beta$ proteins have long been recognized as potent inhibitors of hematopoietic stem and progenitor cell (HSPC) proliferation [9]. This has been clearly demonstrated in earlier in vitro studies where cells, human or murine, are treated with recombinant TGF- $\beta$ (rTGF $\beta 1$ ) in culture. Under these conditions, at single cell level or in batch culture, $\mathrm{rTGF} \beta(300 \mathrm{pg} / \mathrm{mL}$, a concentration found in human plasma [27]) reversibly maintains HSPC cells in a quiescent state without inducing apoptosis [28-30]. Likewise, injection of rTGF $\beta$ directly to mice is sufficient to suppress HSPC proliferation. Conversely, blocking endogenous TGF $\beta$ signaling with neutralizing antibody or antisense oligonucleotides during ex vivo culture promotes HSPC cycling $[31,32]$. The physiological importance of TGF $\beta$ in HSC quiescence was initially challenged with surprising results from mice deficient in TGFBRI. Although TGFBRI-null HSPC have higher proliferative properties than their WT controls in vitro, HSC 


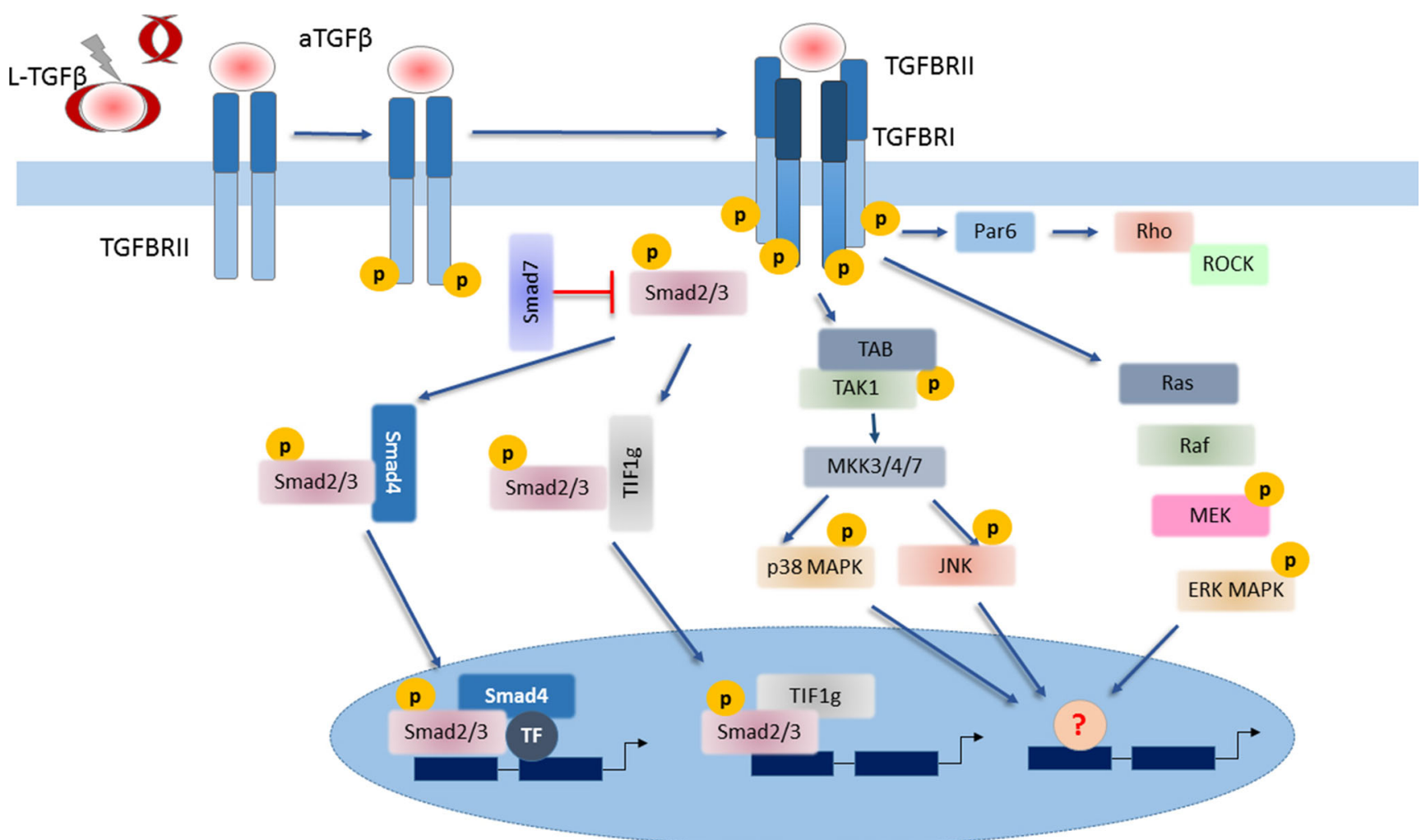

Fig. 1 TGF $\beta$ signaling pathway. TGF $\beta$ factors are secreted as complex inactive protein bound to latency-associated peptide, and the latent TGF $\beta 1$ binding protein-1, called latent TGF $\beta$ (L-TGF $\beta$ ). After dissociation from the complex, TGF $\beta$ binds to membrane-bound type I and II receptors. Upon binding, the constitutively activated type II receptor (TGFBRII) recruits and activates by phosphorylation the type I receptor (TGFBRI), which then activates canonical (smad) or non-

quiescence in vivo is not altered by loss of TGFRI; HSC numbers and repopulation potential from TGFBRI-null mice are conserved, suggesting that perhaps TGF $\beta$ is not essential to maintain HSC quiescence in vivo [33,34]. However, loss of the TGFBRII does cause an increase in HSC cycling in vivo [35]. In other studies, reducing TGF $\beta$ levels in the BM microenvironment and subsequent TGF $\beta$ signaling in HSC, which was achieved by eliminating megakaryocytes - the major source of TGF $\beta$ in the BM in vivo, decreases HSC quiescence by twofold [36•]. In both cases, the permanent suppression of TGF $\beta$ signaling, and subsequent inability to maintain HSC in a quiescent state, lead to a loss of long-term HSC functions and engraftment activity. These findings clearly indicate that TGF $\beta$ is a physiological regulator of HSC quiescence. Interestingly, TGF $\beta$ signaling can be activated in HSC, in a few days following myeloablative chemotherapy, due to an increase in the levels of active TGF $\beta$ in the BM [37•]. TGF $\beta$ signaling picks up on a day when HSC returns to quiescence, and it is necessary for HSC to do so since blocking TGF $\beta$ signaling with neutralizing antibody prolongs HSC cycling. In this model, reducing TGF $\beta$ signaling is associated with enhanced hematopoietic recovery [37•]. Hence, canonical (TAK1-p38 MAPK-JNK) signaling pathways. After activation by phosphorylation, Smad2/3 form a trimer with smad 4 and accumulate in the nucleus where the complex binds to master transcription factors for regulation of gene transcription. Another transcription factor TIF1 $\gamma$ can compete with Smad4 and bind to Smad2/3. Alternatively, TGF $\beta$ can signal via JNK and p38MAPK signaling, ERK ${ }^{\mathrm{MAPK}}$, and small Rho GTPases

TGF $\beta$ is needed for HSC quiescence and for maintaining their long-term functions under homeostasis. But, elevated TGF $\beta$ signaling under regenerative conditions seems to prevent the full regenerative potential of HSC, albeit it is necessary for their return to quiescence. Hence, a transient suppression of TGF $\beta$ signaling during this time may have a beneficial outcome for hematopoietic recovery.

The reasons why loss of TGFBRI does not alter HSC quiescence in vivo remain unclear $[9,33]$. It should be noted that TGFBRI-deficiency causes a lethal inflammatory disorder $[38,39]$, making studies on TGF $\beta$ signaling difficult. We can speculate that since TGFBRII can signal independently of TGFBRI and is more highly expressed on HSC than TGFBRI [29], it is possible that TGFBRI and TGFBRII signal differently in the complex in vivo context and that HSC quiescence depends more on TGFBRII-induced pathways than on those activated by TGFBRI. It is also possible that TGFBRI and TGFBRII are differently used under homeostatic and regenerative conditions. If TGFBRI relays TGF $\beta$ signals only under regenerative conditions, then we would expect TGFBRI loss to enhance HSC regenerative capacity after stress such as bone marrow transplantation. This could be 
examined in serial competitive transplantation using limiting dilution settings in order to quantify HSC self-renewal and their regenerative potential, which was not tested. It would be worthwhile to experimentally test these possibilities.

\section{Dual Role of TGF $\beta$ in Quiescence and Proliferation}

To make matters complex, the anti-proliferative effect of TGF $\beta$ is in fact both cell type-specific and dosagedependent [11]. Although the anti-proliferative effect is consistently seen with high concentration of TGF $\beta$ - i.e., beyond $20 \mathrm{pg} / \mathrm{ml}$, lower concentration promotes HSPC proliferation. Ten $\mathrm{pg} / \mathrm{ml}$ of TGF $\beta$ significantly increases myeloid colony formation $[11,40,41]$. It should be noted that most of the studies on TGF $\beta$ proteins have focused on TGF $\beta 1$. TGF $\beta 2$ seems to have similar dosage effects on HSPC [73]. Moreover, TGF $\beta$ elicits different responses from distinct HSC subtypes. This is specifically important in the current context where it is believed that the HSC pool is composed of a number of HSC subtypes, which have distinct functional potentials. Some HSCs generate a higher myeloid-lymphoid ratio [My-biased $\mathrm{HSC}$, others have a higher lymphoidmyeloid ratio [Ly-biased HSC] [42-44]. These HSC subsets can be delineated based on their expression of the signaling lymphocytic activation molecule [SLAM] family molecule CD150, with CD150+ cells exhibiting increased myeloid output relative to CD150dim HSC [45]. Interestingly, TGF $\beta$ is inhibitory to both populations at high concentration. However, at low concentration, TGF $\beta$ is stimulatory for HSC, whereas it is inhibitory to Ly-HSC, in vitro and in vivo [45]. Two independent studies showed that TGF $\beta$ directly injected to mice stimulates My-HSC and increases production of myeloid cells, while it inhibits the turnover of Ly-HSC [45, 46••] (Fig. 2a).

Overall, TGF $\beta$ seems to have dual and dose-dependent effects on myeloid HSC and myeloid progeny, being inhibitory at high dose but stimulatory at lower dose. The mechanism by which TGF $\beta$ stimulates the growth of myeloid cells is largely unknown. On the other hand, the inhibitory function of TGF $\beta$ on HSC seems to be mediated by cyclin-dependent kinase inhibitor Cdkn1c ( $557^{\mathrm{KIP} 2}$ ) - known to maintain HSC quiescence $[29,47,48]$. This was suggested in in vitro studies showing that while quiescent HSC express high levels of p57, p57 expression disappears upon in vitro cytokine stimulation but is maintained by addition of recombinant TGF $\beta$ [29]. On the other hand cell cycle arrest by TGF $\beta$ requires p 57 expression [47]. In vivo during recovery from myeloablative chemotherapy, p57 expression increases during the window of TGF $\beta$ activation but not in mice that were concomitantly treated with TGF $\beta$-neutralizing antibody, indicating that $\mathrm{p} 57$ is a downstream target of TGF $\beta$ in HSPC [37•]. In addition, TGF $\beta$ can modulate the expression of several classes of cyclin-dependent kinase inhibitor, p15 ${ }^{\mathrm{INK} 4 \mathrm{a}}, \mathrm{p} 21^{\mathrm{Cip} 1}, \mathrm{p} 27^{\mathrm{Kip} 1}$ in hematopoietic cell lineages [49-51]. p21 ${ }^{\text {Cip1 }}$, p2 $7^{\text {Kip1 }}$ are known to inhibit HSCP proliferation [52]. This regulation likely contributes to the proliferative inhibitory effect of TGF $\beta$ on hematopoietic lineages, at least under given conditions, although it seems that TGF $\beta$ can also control HSC quiescence independently of p21 and p27 [53]. The mRNA binding protein Musashi (Msi)-2 may play important roles in TGF $\beta$-dependent lineage biases. Msi2 binds to the 3' untranslated regions of Smad3 and TGFBRI mRNA in HSC. Loss of Msi-2 significantly reduces mRNA expression of TGFBR in HSC, although expression of the protein at the HSC cell surface is increased. Phosphorylation of Smad2/ Smad3 is also reduced in Msi-2-deficient HSC, and so is that of p57. This was associated with loss of HSC quiescence and lineage skewing [54].

\section{Dual Role of TGF $\beta$ Signaling in Young Versus Aged HSC}

The duality of TGF $\beta$ functions is likely physiologically important. The hematopoietic system declines with age. With age, the pool of immunophenotypically defined HSC increases but their biological functions change $[55,56]$. Their self-renewing capacity greatly diminishes with age. In addition, their capacity to generate lymphoid cells declines whereas their propensity to produce myeloid progeny increases. There is compelling evidence to believe that these changes arise, at least in part, from changes in the clonal composition of the HSC pool and perhaps HSC-intrinsic changes. MyHSCs accumulate with age and may contribute to generating a myeloid-biased hematopoietic system in aged mice [45, 57]. As noted above, My-HSC and Ly-HSC population differently respond to TGF $\beta[45,46 \bullet \cdot$. However, this response changes with age. While in young mice $\operatorname{rTGF} \beta$ stimulated My-HSC and promoted myeloid cell development in vivo, rTGF $\beta$ strikingly induced a reduction in myeloid cell production in old mice [46••]. There was also an increased proportion of dormant HSC in the bone marrow of old mice treated with rTGF $\beta$, whereas similar treatment had little effect on the quiescence of young HSC. Interestingly, TGFBRI expression increased in old HSC compared to young HSC, which may explain the sensitivity of old HSC to TGF $\beta$ signaling [46••]. One may envision how TGF $\beta$ contributes to chronological aging. Low TGFBR expression associated with low TGF $\beta$ signaling in young HSC may favor My-HSC and myeloid progeny expansion while reducing Ly-HSC expansion, perhaps contributing to changing the clonal composition of the HSC pool over time. The increasing My-HSC pool may acquire higher TGFBRI expression and TGF $\beta$ signaling - suppressing both their self-renewal ability and their lineage output, albeit myeloid-biased (Fig. 2b). In support of this, the author detected HSC subsets with higher TGFBRI expression, reduced repopulation ability under competitive transplant settings but with myeloid-biased potential [46••]. Interestingly, 
a

Quiescence/Proliferation

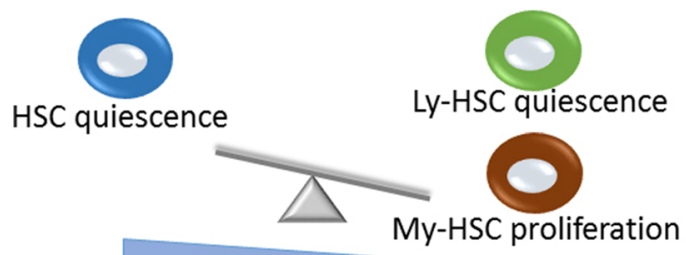

TGF $\beta$

C

\section{Fate decision}

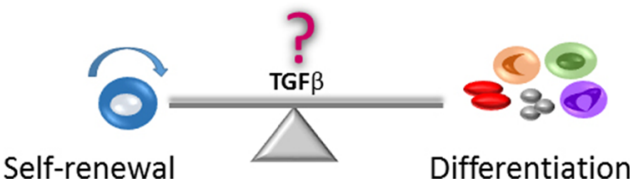

Self-renewal b Aging

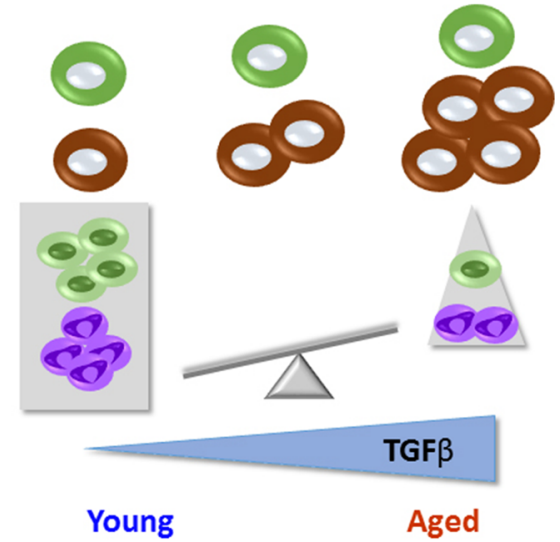

Fig. 2 Multi-faceted role of TGF- $\beta$ signaling in HSCs. a TGF $\beta$ acts in a concentration-dependent manner. At high concentration it induces HSC hibernation / quiescence. At low concentration, TGF $\beta$ promotes proliferation of a myeloid-biased subset of HSCs, whereas it induces quiescence of lymphoid-biased HSC. b Dual role of TGF $\beta$ during aging. rTGF- $\beta$ promotes Myeloid-biased HSC proliferation and myeloid differentiation from young HSC, whereas it induces quiescence in aged HSCs. This duality may contribute to changing the clonal

TGFBRI expression during aging is regulated by Tif1 $\gamma$ (Trim33/Tifl $\gamma$ [tripartite motif family 33/transcription intermediary factor 1 gamma]), a crucial regulator of transcription during hematopoiesis. Tifl $\gamma$ expression is down-regulated in HSC during aging in 20-month old mice compared to 4 month-old mice. Tifl $\gamma$-deficient mice developed an accelerated aging with elevated levels of HSC, a myeloid-biased hematopoiesis, an increase in DNA repair defects, and a shortening of the telomere length seen as early as 4 month-old mice. Tifl $\gamma$-deficient had high expression of TGFBRI and Tifl $\gamma$-deficient HSC were highly sensitive to $\operatorname{rTGF} \beta[46 \bullet \bullet$.

\section{Smad Signaling Pathway}

Most of TGF $\beta$ functions, in particular its inhibitory role on proliferation, are attributed to Smad activation. However, TGF $\beta$ can signal via other pathways, including $\mathrm{p} 38^{\mathrm{MAPK}}$ and RhoA. Since, these pathways are important for HSC functions $[58,59]$, it is reasonable to think that they could mediate some TGF $\beta$ effects. In this context, studies of loss and gain of Smad expression have provided important insights on the specific role of Smads in HSC functions [60].

Studies of enforced expression of Smad 4 in human hematopoietic/progenitor cells from cord blood revealed their growth inhibitory role [61]. Smad 4 overexpression sensitizes composition of the HSC pool during aging causing an accumulation of My-biased HSC and generating a myeloid-biased hematopoietic. c Evidence suggests TGF $\beta$ may control HSC and progenitor fate decisions. Studies in human CD34+ suggest that TGF $\beta$ controls cell fate decisions of myeloid/lymphoid precursors to differentiate into myeloid or lymphoid lineage. Our work suggests that TGF $\beta$ controls HSC fate decisions to self-renewal or to commit to differentiation

HSPC to TGF $\beta$, resulting in their growth arrest and apoptosis in vitro, and a subsequent reduction in their reconstitution capacity in vivo, although their contribution to all blood lineages is intact. Thus, increasing Smad signaling negatively impacts HSC regenerative capacity. Conversely, reducing Smad2/3 activation in murine HSC by means of overexpression of Smad7 prevents the growth inhibitory effect of TGF $\beta$ in vitro, and increases the pool of HSCP [LSK] [62]. This also leads to enhanced HSC regenerative capacity in vivo following transplantation to mice. These findings are consistent with the idea that, under certain stress conditions, increasing TGF $\beta$ signaling in HSC reduces their regenerative capacity in vivo whereas inhibiting TGF $\beta$ signaling promotes it.

The Karlsson's group reported interesting findings on the role of Smad4 in HSC and hematopoiesis using the conditional MxCre/Smad4 Flox/flox mouse line [63]. Smad4 ${ }^{-/-}$HSCs surprisingly possess normal proliferation. Colony and singlecell proliferation assays demonstrated that clonogeneicity and proliferation of hematopoietic progenitors in vitro are unaffected by Smad4 deficiency. Smad $4^{-/-}$HSCs have normal homing to the BM. Further, recipients of transplanted Smad $4^{-/-}$HSCs have normal PB cell counts and mature lineage distribution, suggesting that Smad4 is also dispensable for HSC differentiation. Yet, Smad4 ${ }^{-/}$HSCs exhibit substantial loss of self-renewal ability, as seen in long-term competitive repopulation assays. Under these conditions, recipients of 
$\mathrm{Smad}^{-/-}$BM cells have fourfold lower hematopoietic reconstitution than WT donor cells, although their lineage distribution output remains normal [63]. Intriguingly, these results may be due to lower proliferation capacity of Smad4 ${ }^{-/-}$ HSCs under regenerative conditions, although the difference is very modest. Hence, in this case, a permanent reduction in Smad4 signaling in vivo does not enhance HSC proliferation, as it may have been expected; but it still leads to loss of HSC functions. The finding implies an unsuspected positive role of TGF $\beta$ in HSC functions that may be independent of cell cycle regulation. It is unclear whether the slight difference in proliferation can account for the drastic loss in self-renewal ability Smad $4^{-/}$HSCs. The authors suggest Smad 4 might play a role in the balance between self-renewal and differentiation. It would be interesting to formally test this possibility.

Studies in human HSCP revealed yet another 'twist' to TGF $\beta$ functions. Smad7 overexpression in human HSCP from cord blood causes a shift from lymphoid dominant graft toward increased myeloid contribution, and increases the myeloid-committed clonogenic progenitor frequency [64]. Since both myeloid and B-lymphoid lineages develop normally in these cells, the effect of Smad7 overexpression likely results from a change in cell fate commitment decisions of myeloid/lymphoid precursor by augmenting myeloid differentiation at the expense of lymphoid commitment. The findings strongly suggest TGF $\beta$ signaling modulates the fate decisions of primary multipotent human repopulating cells.

Clearly, studies on Smads reveal that while they mediate TGF $\beta$ effect on HSC quiescence, they also support functions that go well beyond the canonical TGF $\beta$ quiescence axis. It is unclear, though, whether variation in Smad activity mediates the dosage effect of TGF $\beta$. On the other hand, since Smad pathways do not seem to mediate the full range of known TGF $\beta$ effects, non-Smad pathways likely play critical roles in TGF $\beta$ functions, which have been completely overlooked so far.

\section{Beyond Quiescence and Proliferation: Cell Fate and Lineage Differentiation}

\section{Lineage Differentiation}

TGF $\beta$ is a well-known inducer of cell differentiation in various lineages, including the erythroid and myeloid lineages. In the erythroid lineage, TGF $\beta$ facilitates terminal differentiation of already committed progenitors $[65,66]$. When added to cultures containing IL-3, SCF, and EPO, TGF $\beta$ stimulated the rapid appearance of hemoglobin-positive cells from CD36+ erythroid progenitors due to an early induction of erythroid differentiation genes. Conversely, it inhibited immature progenitor proliferation such that the known reduction of BFU-E numbers by TGF $\beta$ may be due to a 'conversion' of
BFU-E into CFU-E by skipping cell divisions $[65,66]$. As such, TGF $\beta$ exposure in vivo results in ineffective erythropoiesis. Noteworthy, TGF $\beta$ signaling is elevated in hematopoietic cells of patients diagnosed with myelodysplastic syndromes (MDS) $[67,68]$. The ineffective erythropoiesis seen in MDS can be alleviated by blocking the TGF $\beta$ pathway using a small molecule inhibitor or TGF $\beta$-family ligand trap [67-69]. Interestingly, the dual effect of TGF $\beta$ on progenitor proliferation and erythroid differentiation are controlled by distinct branches of the TGF $\beta$ pathway, Smad4 and TIF $1 \gamma$, respectively. In erythroid cells, TIF $1 \gamma$ can bind to R-Smad2/3 in competition with Smad4 in response to TGF $\beta$ and mediates the erythroid differentiation response, whereas Smad 4 mediates the antiproliferative response [23].

TGF $\beta$ exhibits a contrasting effect in the myeloid lineage by promoting granulopoiesis in the presence of cytokines. In in vitro studies, TGF $\beta$ stimulates GM-CSF-induced myeloid progenitor frequency and neutrophilic differentiation [70]. Administration of TGF $\beta$ in vivo increases total numbers of CFU-GM and myeloblasts per femur and enhances numbers of mature granulocytes in both the bone marrow and peripheral blood of young mice [71]. TGF $\beta$ is also a master regulator of lymphoid differentiation, but this will not be discussed here.

\section{Regulation of Cell Identity by TGF $\beta$ Signaling}

Overall, these findings demonstrate the plurality and complexity of TGF $\beta$ functions in the hematopoietic system whereby TGF $\beta$ elicits different responses in different cell types. Recently, two elegant studies provided insights on how the Smads are used by the cells to regulate different fates [72, 73]. During lineage differentiation, $\operatorname{Smad} 3$ is recruited by master transcription factors (TF) to DNA sites by establishing open chromatin containing Smad-binding elements allowing Smad to co-occupy the genome and form a complex with celltype-specific master TF at target genes. In turn, Smad recruits co-factors to occupy the same DNA sites forming a corecomplex that determines expression of lineage identity genes. This circuit instructs cell identity since expression of a master TF in cells in which it is not normally expressed can redirect Smad3 binding to sites newly occupied by this TF. This cooperation has been shown in hematopoietic and nonhematopoietic lineages. Smad1 cooperates with $\mathrm{C} / \mathrm{EBP} \alpha$ for myeloid lineage regulation, whereas it interacts with GATA1 in erythroid cells [73]. Smad3 occupies the genomes with PU.1 in pro-B cells [72]. Induction of $\mathrm{C} / \mathrm{EBP} \alpha$ in erythroid cells shifted Smad1 binding to sites occupied by $\mathrm{C} / \mathrm{EBP} \alpha$ [73]. Hence, Smads serve as co-factors of master TF used by a given cell type for maintaining its identity and regulating its function. In this context, it is easy to envision that small changes in Smad, disrupting this equilibrium, may alter cell fate. The outcome of TGF $\beta$ deregulation will thus depend on the cell type and its stage of differentiation. 


\section{TGF $\beta$ and HSC Fate Decisions?}

How HSCs use TGF $\beta$ still remains ill-defined. In HSC, TGF $\beta$ is mostly viewed as a regulator of quiescence. However, TGF $\beta$ actions are likely not limited to quiescence. In fact, as noted previously TGF $\beta$ may be important to modulate HSC identity and fate decisions. Interestingly, Smad4-null HSC had significantly reduced long-term repopulation activity and selfrenewal compared to WT cells; yet, cell cycle status and apoptosis were unaffected by Smad4 deletion, strongly suggesting cell cycle-independent roles [63]. Smad7 overexpression likely induces a change in cell fate commitment decisions of myeloid/lymphoid precursors [64]. TIF1 $\gamma$ appears to modulate the PU.1/GATA1 antagonism paradigm to modulate erythroid versus myeloid fate decisions from HSC [74]. Hence, TGF $\beta$ could be a non-recognized master regulator of HSPC fate decision at various branching points of the hematopoietic tree.

Interestingly, our lab has shown that TGF $\beta$ signaling increases following bone marrow transplantation and, consistent with other studies, this increase limits HSC self-renewal and regenerative capacity. Indeed, we could show that inhibiting TGF $\beta$ during regeneration improved long-term HSC engraftment. Yet, the negative effect of TGF $\beta$ on HSC functions was not linked to HSC quiescence. Instead, TGF $\beta$ was involved in the decision-making of HSC to self-renew or to commit to differentiation, as seen by the fact that increasing TGF $\beta$ signaling in multipotent HSPC reduced their ability to generate daughter cells that retain multilineage potential, [examined in the so-called paired-daughter cell assay], although their division kinetics was not altered. Most interestingly, in this case, TGF $\beta$ was secreted by HSC themselves, which reinforces the underestimated importance of TGF $\beta$ autocrine signaling in HSC functions, and its effect was mediated by the noncanonical $\mathrm{p} 38^{\mathrm{MAPK}}$ pathway. These findings require further investigation, but support the notion that TGF $\beta$ participates in HSC fate decisions independent of cell cycle regulation, at least under regenerative conditions following bone marrow transplantation [72].

\section{TGF $\beta$ Regulation: Source of TGF $\beta$}

\section{The Role of the BM Microenvironment}

In the hematopoietic system, latent TGF $\beta$ is highly expressed in megakaryocytes (MK) [36•], and is abundant in mineralized bones [75]. Likewise, platelets are considered to be the most abundant reservoir of TGF $\beta$ in the PB. Two independent groups have reported that $\mathrm{MK}$ are important for maintaining HSC quiescence under homeostatic conditions $[36 \bullet, 76]$. Zhao et al. further showed that MKs maintain HSC quiescence through TGF $\beta$ signaling [36•]. In immunostained bone marrow sections, the authors showed that HSC with nuclear localization of $\mathrm{p}$-smad - indicative of activation of TGF $\beta$ signaling - are adjacent to MK in vivo. In MK-depleted mice, using Pf4-cre;iDTR mice, TGF $\beta$ protein expression is greatly reduced and so is TGF $\beta$ biological activity, as assessed by the luciferase reporter assay. The numbers of p-smad+ HSC are reduced by MK depletion, and these HSCs are released from quiescence. Finally, deletion of TGF $\beta$ specifically in MK results in HSC cycling [36•]. How TGF $\beta$ is releasing from the latent complex in this model remains to be examined. Interestingly, an independent study revealed the unexpected role of non-myelinated Schwann cells [35]. Anti-TGF $\beta$ antibody that specifically recognizes TGF $\beta$ only after its release from the latent TGF $\beta$ complex and anti-LAP antibody revealed differential patterns of expression of latent TGF $\beta$ and active TGF $\beta$ in BM section. LAP is detected in various cells, including in MK, whereas distribution of active TGF $\beta$ is highly restricted to a minor population of LAP-positive cells that form long and spindled structures and was identified as nonmyelinated Schwann cells - cells of the peripheral nervous system [35]. These cells express the integrin beta 8 (Itgb8), which likely contributes to processing latent TGF $\beta$ into active TGF $\beta$. The functional significance of these findings is further supported in studies in which the lumbar sympathetic trunk was cut to cause denervation of sympathetic nerves. It reduced the number of cells producing active TGF $\beta$ and resulted in loss of HSC and their repopulation ability, underscoring that Schwann cells constitute an important component of the HSC niche and maintain HSC hibernation by regulating activation of latent TGF $\beta$ [35] (Fig. 3). Whether similar cells/ mechanisms participate in TGF $\beta$ activation under regenerative hematopoietic stress conditions remains to be seen.

\section{Endogenous TGF $\beta$}

TGF $\beta$ can also be secreted by HSC themselves, and exerts autocrine effects on HSC functions [12]. These effects are best documented by the observation that pharmacological inhibitors of TGF $\beta$ signaling, TGF $\beta$ oligonucleotide antisense, expression of a dominant negative mutant of TGFBRII or TGFTGF $\beta$-neutralizing antibody can reduce TGF $\beta$ signaling in HSCP and increase the cycling of HSC in vitro culture under serum-free conditions in the presence of cytokines, SCF, and TPO alone [31, 32, 77, 78]. Unfortunately, there is little information on the role of endogenous TGF $\beta$ in HSC functions. Half of the mice lacking TGF $\beta 1$ die in utero due to defects in vasculogenesis and hematopoiesis [79]. The remaining adult TGF $\beta 1$-null mice developed lethal inflammatory disorders within a few days of age, making the study of TGF $\beta$ during adulthood impossible $[79,80]$. Since the inflammatory disease is not transplantable after T-cell depletion, information on engraftment ability of bone marrow cells from 8-10 days-old neonates (before the inflammatory disease develops) and of 


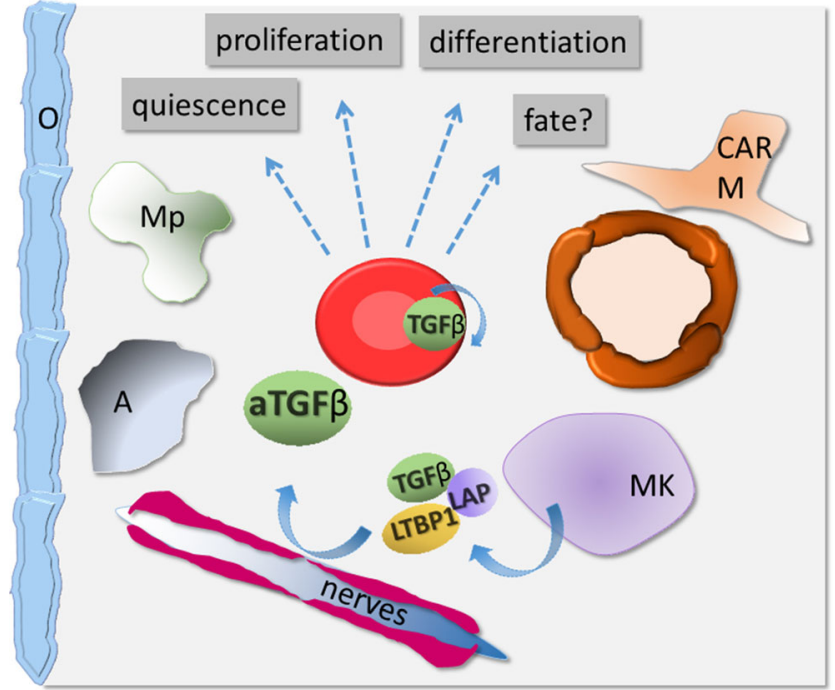

Fig. 3 Activation of TGF $\beta$ signaling and its role in HSC function. TGF $\beta$ is secreted as an inactive complex bound to latency-associated peptide (LAP) and the latent TGF $\beta 1$ binding protein-1 (LTBP1) by megakaryocytes. Non-myelinated Schwann cells contribute to releasing the biologically active form of TGF $\beta$ from the latent complex. Active TGF $\beta$ can exert multiple functions on HSC and progenitors, including quiescence, proliferation, differentiation, and perhaps fate decisions

embryonic day 14 fetal liver could be obtained and showed impaired short- and long-term repopulation activity, which may be due to HSC homing defects to the BM [81]. In vitro studies indicated impaired survival of TGF $\beta$-null HSPC [81]. To date, there are no published studies that have used conditional TGF $\beta$-Flox-Flox mice to gain insights in TGF $\beta$ effects on adult hematopoiesis. Nonetheless, these findings clearly underscore the functional importance of endogenous TGF $\beta$ in hematopoiesis. This requires further investigation to understand in detail how TGF $\beta$ works.

\section{Conclusions}

TGF $\beta$ clearly is a key factor of HSC quiescence and a suppressor of lineage development, and the Smad pathway largely mediates this effect. It is also clear that TGF $\beta$ plays critical roles beyond quiescence, in cell identity and perhaps in cell fate decisions at each branching point of $\mathrm{HSC}$ and multipotent progenitor fate decisions. How HSC uses TGF $\beta$ signaling in doing so remains to be fully understood. Which signaling pathways mediates TGF $\beta$ function in HSC, do the Smads mediate TGF $\beta$ dosage effect, which branches of the TGF $\beta$ pathways are triggered by autocrine TGF $\beta$ and what are the roles of non-smad pathways remain to be investigated. Blocking TGF $\beta$ signaling pathways during the regenerative phase of hematopoiesis following injury due to chemotherapy or bone marrow transplantation may improve blood cell recovery. However, components of the TGF $\beta$ pathway are often found mutated or deregulated in a variety of diseases. Loss of
TGF $\beta$ is often associated with leukemia. Conversely, upregulation of TGF $\beta$ pathway in hematopoietic cells or in the microenvironment participates in myelodysplastic syndromes, myelofibrosis, and myeloproliferative disorders. Therefore, understanding further how TGF $\beta$ works in hematopoiesis is important not only for our fundamental understanding of HSC biology but also for therapeutic purposes.

Acknowledgments The work was supported by NIH (American Society of Hematology Bridge award to-MDF; R01 DK102890 to MDF).

\section{Compliance with Ethical Standards}

Conflict of Interest Ashwini Hinge declares no conflict of interest. Marie-Dominique Filippi reports an issued patent 9334284.

Human and Animal Rights and Informed Consent This article does not contain any studies with human or animal subjects performed by any of the authors.

\section{References}

Papers of particular interest, published recently, have been highlighted as:

- Of importance

•. Of major importance

1. Orkin SH, Zon LI. Hematopoiesis: an evolving paradigm for stem cell biology. Cell. 2008;132(4):631-44.

2. Zon LI. Intrinsic and extrinsic control of haematopoietic stem-cell self-renewal. Nature. 2008;453(7193):306-13.

3. Wilson A, Laurenti E, Trumpp A. Balancing dormant and selfrenewing hematopoietic stem cells. Curr Opin Genet Dev. 2009;19(5):461-8.

4. Morrison SJ, Kimble J. Asymmetric and symmetric stem-cell divisions in development and cancer. Nature. 2006;441(7097): 1068-74.

5. Anthony BA, Link DC. Regulation of hematopoietic stem cells by bone marrow stromal cells. Trends Immunol. 2014;35(1):32-7.

6. Birbrair A, Frenette PS. Niche heterogeneity in the bone marrow. Ann N Y Acad Sci. 2016;1370(1):82-96.

7. Yu VW, Scadden DT. Hematopoietic stem cell and its bone marrow niche. Curr Top Dev Biol. 2016;118:21-44.

8. Massague J. TGFbeta signalling in context. Nat Rev Mol Cell Biol. 2012;13(10):616-30.

9. Blank U, Karlsson S. TGF-beta signaling in the control of hematopoietic stem cells. Blood. 2015;125(23):3542-50.

10. Oshimori N, Fuchs E. The harmonies played by TGF-beta in stem cell biology. Cell Stem Cell. 2012;11(6):751-64.

11. Soderberg SS, Karlsson G, Karlsson S. Complex and context dependent regulation of hematopoiesis by TGF-beta superfamily signaling. Ann N Y Acad Sci. 2009;1176:55-69.

12. Ruscetti FW, Akel S, Bartelmez SH. Autocrine transforming growth factor-beta regulation of hematopoiesis: many outcomes that depend on the context. Oncogene. 2005;24(37):5751-63.

13. Massague J, Gomis RR. The logic of TGFbeta signaling. FEBS Lett. 2006;580(12):2811-20. 
14. Attisano L, Wrana JL. Signal integration in TGF-beta, WNT, and Hippo pathways. F1000Prime Rep. 2013;5:17.

15. Annes JP, Munger JS, Rifkin DB. Making sense of latent TGFbeta activation. J Cell Sci. 2003;116(Pt 2):217-24.

16. Koli K et al. Latency, activation, and binding proteins of TGF-beta. Microsc Res Tech. 2001;52(4):354-62.

17. Munger JS et al. Latent transforming growth factor-beta: structural features and mechanisms of activation. Kidney Int. 1997;51(5): 1376-82.

18. Murphy-Ullrich JE, Poczatek M. Activation of latent TGF-beta by thrombospondin-1: mechanisms and physiology. Cytokine Growth Factor Rev. 2000;11(1-2):59-69.

19. Munger JS et al. The integrin alpha $\mathrm{v}$ beta 6 binds and activates latent TGF beta 1: a mechanism for regulating pulmonary inflammation and fibrosis. Cell. 1999;96(3):319-28.

20. Barcellos-Hoff MH, Dix TA. Redox-mediated activation of latent transforming growth factor-beta 1. Mol Endocrinol. 1996;10(9): 1077-83.

21. Jobling MF et al. Isoform-specific activation of latent transforming growth factor beta (LTGF-beta) by reactive oxygen species. Radiat Res. 2006;166(6):839-48.

22. Mu Y, Gudey SK, Landstrom M. Non-Smad signaling pathways. Cell Tissue Res. 2012;347(1):11-20.

23. He W et al. Hematopoiesis controlled by distinct TIF1gamma and Smad4 branches of the TGFbeta pathway. Cell. 2006;125(5):929-41.

24. Tang $\mathrm{M}$ et al. TAK1 is required for the survival of hematopoietic cells and hepatocytes in mice. J Exp Med. 2008;205(7):1611-9.

25. Yamaguchi $\mathrm{K}$ et al. Identification of a member of the MAPKKK family as a potential mediator of TGF-beta signal transduction. Science. 1995;270(5244):2008-11.

26. Guo X, Wang XF. Signaling cross-talk between TGF-beta/BMP and other pathways. Cell Res. 2009;19(1):71-88.

27. Junker $U$ et al. Transforming growth factor beta 1 is significantly elevated in plasma of patients suffering from renal cell carcinoma. Cytokine. 1996;8(10):794-8.

28. Batard P et al. TGF-(beta) 1 maintains hematopoietic immaturity by a reversible negative control of cell cycle and induces CD34 antigen up-modulation. J Cell Sci. 2000;113(Pt 3):383-90.

29. Yamazaki $\mathrm{S}$ et al. TGF-beta as a candidate bone marrow niche signal to induce hematopoietic stem cell hibernation. Blood. 2009;113(6):1250-6.

30. Sitnicka $\mathrm{E}$ et al. Transforming growth factor beta 1 directly and reversibly inhibits the initial cell divisions of long-term repopulating hematopoietic stem cells. Blood. 1996;88(1):82-8.

31. Cardoso AA et al. Release from quiescence of CD34+ CD38- human umbilical cord blood cells reveals their potentiality to engraft adults. Proc Natl Acad Sci U S A. 1993;90(18):8707-11.

32. Hatzfeld $\mathrm{J}$ et al. Release of early human hematopoietic progenitors from quiescence by antisense transforming growth factor beta 1 or Rb oligonucleotides. J Exp Med. 1991;174(4):925-9.

33. Larsson $\mathrm{J}$ et al. TGF-beta signaling-deficient hematopoietic stem cells have normal self-renewal and regenerative ability in vivo despite increased proliferative capacity in vitro. Blood. 2003;102(9): 3129-35.

34. Larsson J et al. Quiescence of hematopoietic stem cells and maintenance of the stem cell pool is not dependent on TGF-beta signaling in vivo. Exp Hematol. 2005;33(5):592-6.

35. Yamazaki $\mathrm{S}$ et al. Nonmyelinating Schwann cells maintain hematopoietic stem cell hibernation in the bone marrow niche. Cell. 2011;147(5):1146-58

36. Zhao $\mathrm{M}$ et al. Megakaryocytes maintain homeostatic quiescence and promote post-injury regeneration of hematopoietic stem cells. Nat Med. 2014;20(11):1321-6. This paper provides definitive evidence that TGF-beta secreted by megakaryocytes controls hematopoietic stem cell quiescence in vivo.
37. Brenet $\mathrm{F}$ et al. TGFbeta restores hematopoietic homeostasis after myelosuppressive chemotherapy. J Exp Med. 2013;210(3):623-39. This study convincingly shows that TGF-beta limits the regenerative capacity of hematopoietic stem and progenitor cells following myeloablative therapy; but it helps HSC to return into quiescence after stress in vivo.

38. Leveen $\mathrm{P}$ et al. Induced disruption of the transforming growth factor beta type II receptor gene in mice causes a lethal inflammatory disorder that is transplantable. Blood. 2002;100(2):560-8.

39. Yaswen $\mathrm{L}$ et al. Autoimmune manifestations in the transforming growth factor-beta 1 knockout mouse. Blood. 1996;87(4):1439-45.

40. Kale VP. Differential activation of MAPK signaling pathways by TGF-betal forms the molecular mechanism behind its dosedependent bidirectional effects on hematopoiesis. Stem Cells Dev. 2004;13(1):27-38.

41. Kale VP, Vaidya AA. Molecular mechanisms behind the dosedependent differential activation of MAPK pathways induced by transforming growth factor-beta1 in hematopoietic cells. Stem Cells Dev. 2004;13(5):536-47.

42. Sieburg HB et al. The hematopoietic stem compartment consists of a limited number of discrete stem cell subsets. Blood. 2006;107(6): 2311-6.

43. Benz $\mathrm{C}$ et al. Hematopoietic stem cell subtypes expand differentially during development and display distinct lymphopoietic programs. Cell Stem Cell. 2012;10(3):273-83.

44. Dykstra B et al. Long-term propagation of distinct hematopoietic differentiation programs in vivo. Cell Stem Cell. 2007;1(2):218-29.

45. Challen GA et al. Distinct hematopoietic stem cell subtypes are differentially regulated by TGF-beta1. Cell Stem Cell. 2010;6(3):265-78.

46.• Quere $\mathrm{R}$ et al. Tifl gamma regulates the TGF-betal receptor and promotes physiological aging of hematopoietic stem cells. Proc Natl Acad Sci U S A. 2014;111(29):10592-7. This study thoroughly dissects the role of TGF-beta1 and its regulator Tiflgamma in young versus aged hematopoietic stem cells.

47. Scandura JM et al. Transforming growth factor beta-induced cell cycle arrest of human hematopoietic cells requires p57KIP2 upregulation. Proc Natl Acad Sci U S A. 2004;101(42):15231-6.

48. Yamazaki $\mathrm{S}$ et al. Cytokine signals modulated via lipid rafts mimic niche signals and induce hibernation in hematopoietic stem cells. Embo J. 2006;25(15):3515-23.

49. Dao MA, Hwa J, Nolta JA. Molecular mechanism of transforming growth factor beta-mediated cell-cycle modulation in primary human CD34(+) progenitors. Blood. 2002;99(2):499-506.

50. Dao MA, Taylor N, Nolta JA. Reduction in levels of the cyclindependent kinase inhibitor p27(kip-1) coupled with transforming growth factor beta neutralization induces cell-cycle entry and increases retroviral transduction of primitive human hematopoietic cells. Proc Natl Acad Sci U S A. 1998;95(22):13006-11.

51. Ducos K et al. p21(cip1) mRNA is controlled by endogenous transforming growth factor-beta1 in quiescent human hematopoietic stem/progenitor cells. J Cell Physiol. 2000;184(1):80-5.

52. Orford KW, Scadden DT. Deconstructing stem cell self-renewal: genetic insights into cell-cycle regulation. Nat Rev Genet. 2008;9(2):115-28.

53. Cheng $\mathrm{T}$ et al. Transforming growth factor beta 1 mediates cellcycle arrest of primitive hematopoietic cells independent of p21(Cip1/Waf1) or p27(Kip1). Blood. 2001;98(13):3643-9.

54. Park SM et al. Musashi-2 controls cell fate, lineage bias, and TGFbeta signaling in HSCs. J Exp Med. 2014;211(1):71-87.

55. Beerman I et al. Stem cells and the aging hematopoietic system. Curr Opin Immunol. 2010;22(4):500-6.

56. Geiger H, Denkinger M, Schirmbeck R. Hematopoietic stem cell aging. Curr Opin Immunol. 2014;29:86-92.

57. Beerman I et al. Functionally distinct hematopoietic stem cells modulate hematopoietic lineage potential during aging by a 
mechanism of clonal expansion. Proc Natl Acad Sci U S A. 2010;107(12):5465-70.

58. Ito $\mathrm{K}$ et al. Reactive oxygen species act through p38 MAPK to limit the lifespan of hematopoietic stem cells. Nat Med. 2006;12(4):446-51.

59. Zhou X et al. RhoA GTPase controls cytokinesis and programmed necrosis of hematopoietic progenitors. J Exp Med. 2013;210(11): 2371-85.

60. Utsugisawa $\mathrm{T}$ et al. A road map toward defining the role of Smad signaling in hematopoietic stem cells. Stem Cells. 2006;24(4): 1128-36.

61. Rorby E et al. Human hematopoietic stem/progenitor cells overexpressing Smad4 exhibit impaired reconstitution potential in vivo. Blood. 2012;120(22):4343-51.

62. Blank U et al. Smad7 promotes self-renewal of hematopoietic stem cells. Blood. 2006;108(13):4246-54.

63. Karlsson $\mathrm{G}$ et al. Smad4 is critical for self-renewal of hematopoietic stem cells. J Exp Med. 2007;204(3):467-74.

64. Chadwick K et al. Smad7 alters cell fate decisions of human hematopoietic repopulating cells. Blood. 2005;105(5):1905-15.

65. Krystal G et al. Transforming growth factor beta 1 is an inducer of erythroid differentiation. J Exp Med. 1994;180(3):851-60.

66. Zermati $\mathrm{Y}$ et al. Transforming growth factor inhibits erythropoiesis by blocking proliferation and accelerating differentiation of erythroid progenitors. Exp Hematol. 2000;28(8):885-94.

67. Zhou L et al. Reduced SMAD7 leads to overactivation of TGF-beta signaling in MDS that can be reversed by a specific inhibitor of TGF-beta receptor I kinase. Cancer Res. 2011;71(3):955-63.

68. Zhou L et al. Inhibition of the TGF-beta receptor I kinase promotes hematopoiesis in MDS. Blood. 2008;112(8):3434-43.

69. Suragani RN et al. Transforming growth factor-beta superfamily ligand trap ACE-536 corrects anemia by promoting late-stage erythropoiesis. Nat Med. 2014;20(4):408-14.

70. Hestdal $\mathrm{K}$ et al. Increased granulopoiesis after sequential administration of transforming growth factor-beta 1 and granulocyte- macrophage colony-stimulating factor. Exp Hematol. 1993;21(6): 799-805.

71. Keller JR et al. Stimulation of granulopoiesis by transforming growth factor beta: synergy with granulocyte/macrophage-colonystimulating factor. Proc Natl Acad Sci U S A. 1991;88(16):7190-4.

72. Mullen AC et al. Master transcription factors determine cell-typespecific responses to TGF-beta signaling. Cell. 2011;147(3): 565-76.

73. Trompouki E et al. Lineage regulators direct BMP and Wnt pathways to cell-specific programs during differentiation and regeneration. Cell. 2011;147(3):577-89.

74. Monteiro R, Pouget C, Patient R. The gata1/pu.1 lineage fate paradigm varies between blood populations and is modulated by tifl gamma. EMBO J. 2011;30(6):1093-103.

75. Wu M, Chen G, Li YP. TGF-beta and BMP signaling in osteoblast, skeletal development, and bone formation, homeostasis and disease. Bone Res. 2016;4:16009.

76. Bruns I et al. Megakaryocytes regulate hematopoietic stem cell quiescence through CXCL4 secretion. Nat Med. 2014;20(11): 1315-20.

77. Fan $\mathrm{X}$ et al. Transient disruption of autocrine TGF-beta signaling leads to enhanced survival and proliferation potential in single primitive human hemopoietic progenitor cells. J Immunol. 2002;168(2):755-62.

78. Soma T, Yu JM, Dunbar CE. Maintenance of murine long-term repopulating stem cells in ex vivo culture is affected by modulation of transforming growth factor-beta but not macrophage inflammatory protein-1 alpha activities. Blood. 1996;87(11):4561-7.

79. Christ $\mathrm{M}$ et al. Immune dysregulation in TGF-beta 1-deficient mice. J Immunol. 1994;153(5):1936-46.

80. Letterio JJ et al. Autoimmunity associated with TGF-beta1deficiency in mice is dependent on MHC class II antigen expression. J Clin Invest. 1996;98(9):2109-19.

81. Capron $\mathrm{C}$ et al. A major role of TGF-beta1 in the homing capacities of murine hematopoietic stem cell/progenitors. Blood. 2010;116(8): 1244-53. 